\title{
Optimizing the immobilization of gold nanoparticles on functionalized silicon surfaces: amine- vs thiol-terminated silane
}

\author{
Maroua Ben Haddada • Juliette Blanchard • \\ Sandra Casale • Jean-Marc Krafft • Anne Vallée • \\ Christophe Méthivier • Souhir Boujday
}

Published online: 17 November 2013

(C) The Author(s) 2013. This article is published with open access at SpringerLink.com

\begin{abstract}
Immobilization of gold nanoparticles on planar surfaces is of great interest to many scientific communities; chemists, physicists, biologists, and the various communities working at the interfaces between these disciplines. Controlling the immobilization step, especially nanoparticles dispersion and coverage, is an important issue for all of these communities. We studied the parameters that can influence this interaction, starting with the nature of the terminal chemical function. Thus, we have carefully grafted silanes terminated by either amine or thiol groups starting from aminopropyltriethoxysilane (APTES) or mercaptopropyltriethoxysilane. We also changed the chain length for thiol-terminated layers through covalent grafting of mercaptoundecanoic acid (MUA) on APTESmodified layers, and the protocol of nanoparticles deposition to evaluate whether other factors must be taken into consideration to rationalize this interaction. The formed layers were characterized by X-ray photoelectron spectroscopy and gold nanoparticles deposition was monitored by scanning electron microscopy and surface-enhanced Raman scattering. We observed significant differences in terms of nanoparticles dispersion and density depending on the nature of the chemical layer on silicon. The use of ultrasounds during the deposition process
\end{abstract}

Electronic supplementary material The online version of this article (doi:10.1007/s13404-013-0120-y) contains supplementary material, which is available to authorized users.

M. Ben Haddada · J. Blanchard · S. Casale · J.-M. Krafft •

A. Vallée $\cdot$ C. Méthivier $\cdot$ S. Boujday

UPMC Univ Paris 6, UMR CNRS 7197, Laboratoire de Réactivité de

Surface, 75005 Paris, France

M. Ben Haddada $\cdot$ J. Blanchard $\cdot$ S. Casale $\cdot$ J.-M. Krafft •

A. Vallée $\cdot$ C. Méthivier $\cdot$ S. Boujday $(\triangle)$

Laboratoire de Réactivité de Surface, UMR CNRS 7197, Université

Pierre et Marie Curie-Paris VI, 4 Place Jussieu, 75252 Paris cedex 05, France

e-mail: souhir.boujday@upmc.fr was very efficient to limit aggregates formation. The optimal deposition procedures were obtained through the use of APTES and APTES/MUA functionalization. They were compared in terms of coverage, dispersion, and densities of isolated nanoparticles. The APTES/MUA surfaces clearly showed better results that may arise from both the longer chain and the dilution of thiol end groups.

Keywords Gold nanoparticles · Silicon surface · Silane grafting $\cdot$ Surface functionalization $\cdot$ XPS $\cdot$ Scanning electron microscopy

\section{Introduction}

The fabrication of solid substrates with gold nanoparticles assemblies immobilized on their surface is currently the subject of growing interest because of the key role these substrates could play in the development of several devices [1-3]. The physical and chemical properties of these devices will depend not only on the size and shape of the gold nanoparticles but also on their spatial arrangement and on the nature of their interaction with the substrate surface [4-6]. Biosensors represent one of the areas for which the use of nanoparticles is booming. Indeed, biosensors are analytical tools whose effectiveness is highly dependent on the accuracy of the measurement and thus on its reproducibility [7, 8]. Input from gold nanoparticles in this area is twofold: on the one hand, they allow amplification of the signal transduction for many techniques and, on the other hand, they can provide surface nanostructuration $[9,10]$. In both cases, achieving a densely packed layer and a regular arrangement is crucial to ensure an optimal amplification while preserving a reproducible and quantitative response of transduction techniques [11, 12]. 
Usually, the anchoring of gold nanoparticles on the solid substrates is carried out by using an intermediate layer of organic molecules grafted on the solid surface, whose terminal functional groups are selected for their electrostatic or chemical interactions with the nanoparticles [11-16]. These platforms should also be stable over time and the interaction of the nanoparticles with the surface should be strong enough to ensure that the nanoparticles remains attached to the surface during further functionalization and upon utilization. The molecules commonly used for the functionalization of oxidized silicon wafer or glass slides are organosilane with up to three hydrolysable groups (either -OR or $-\mathrm{Cl}$ ) that would react with the silanols groups from the substrate surface and (at least) a nonhydrolysable group bearing the terminal function responsible for the interaction with the gold nanoparticles [17]. Among the large variety of available organosilanes, aminopropyltriethoxysilane (APTES; $\left.\mathrm{NH}_{2}\left(\mathrm{CH}_{2}\right)_{3} \mathrm{Si}\left(\mathrm{OCH}_{2} \mathrm{CH}_{3}\right)_{3}\right)$ is, by far, the most studied surface modifier (references [18-21] and references therein). APTES is relatively easy to handle thanks to its moderate reactivity. Its three hydrolysable ethoxy groups ensure a robust anchoring of the silane to the surface (silanization step), whereas the amine function of the aminopropyl group remains available for further reaction. Terminal amine functions are extensively used for surface functionalization because they easily react with acid, aldehyde, or thiocyanates through covalent bond. Moreover, amine groups have affinity to gold nanoparticles and are widely used to immobilize them through electrostatic interactions $[4,11,14]$.

The numerous studies dealing with the functionalization of silica/oxidized silicon surface with APTES have shown that the silanization step is very sensitive toward experimental conditions and that the final surface state of the modified substrate depends strongly on temperature, presence of water, concentration of APTES, and the duration of the grafting step [18].

In this work, we studied the organic layer influence on the grafting of gold nanoparticles on functionalized silicon substrates. Our aim was to find the optimal experimental conditions for both an optimal density and dispersion of gold nanoparticles on silicon surfaces. For this purpose, we explored some of the parameters that can influence this interaction, starting with the nature of the terminal chemical function. In a first step, APTES was used to form amine-terminated silane layers. Then, starting from mercaptopropyltriethoxysilane (MPTES), thiol-terminated silane layers were constructed. We also changed the chain length for thiol-terminated layers through covalent grafting of mercaptoundecanoic acid (MUA) on APTES-modified layers. This latter functionalization procedure was successfully used by Kaminska et al. to immobilize CTAB-covered nanoparticles on silicon and reach a densely packed layer [22]. X-ray photoelectron spectroscopy (XPS) and contact angle measurements were used to characterize the formed layers and the protocol of nanoparticle deposition was modified, particularly using ultrasonication during the deposition of the spherical gold nanoparticles. The grafting of nanoparticles was monitored by surfaceenhanced Raman scattering (SERS). Finally, scanning electron microscopy (SEM) was used to compare gold nanoparticles coverage and dispersion for the considered surface functionalizations.

\section{Experimental section}

\section{Materials}

$N$-ethyl- $N$ '-(3-(dimethylamino) propyl) carbodiimide hydrochloride (EDC; $98 \%$ ), sodium citrate ( $\mathrm{HOC}(\mathrm{COONa})$ $\left(\mathrm{CH}_{2} \mathrm{COONa}\right)_{2} \cdot 2 \mathrm{H}_{2} \mathrm{O} ; 99 \%$ ), and gold(III)chloride trihydrate $\left(\mathrm{HAuCl}_{4} \cdot \mathrm{H}_{2} \mathrm{O} ; \geq 99.9 \%\right.$ ) were purchased from Sigma Aldrich. $N$-hydroxysuccinimide (NHS; $97 \%$ ), 11-mercaptoundecanoic acid (MUA; $95 \%$ ), (3-aminopropyl)triethoxysilane (APTES; $99 \%$ ) and (3-mercaptopropyl)trimethoxysilane (MPTES; $95 \%$ were purchased from Aldrich and ethylenediamine (EDA; $\geq 99.5 \%$ ) from Fluka. Silicon wafer $<100>$ from Sigma Aldrich was cut into $1 \times 1 \mathrm{~cm}^{2}$ pieces. Sulfuric acid, $96 \%$ $\left(\mathrm{H}_{2} \mathrm{SO}_{4}\right)$ and hydrogen peroxide, $30 \%$ in water $\left(\mathrm{H}_{2} \mathrm{O}_{2}\right)$ were supplied by Carlo Erba. Toluene, acetone, and ethanol were purchased from Analar Normapur. MilliQ water (18 M $\Omega$, Millipore, France) was used for the preparation of the solutions and for all rinses. All chemicals were reagent grade or higher and were used without further purification.

\section{Surface chemistry}

First, silicon surfaces were cleaned following a procedure which includes several washing step, a treatment with a piranha solution, and finally a treatment with UV ozone. A detailed description of this procedure is given in reference [18].

The same experimental conditions were applied for silane grafting for the amine-terminated silane (APTES) and for the thiol-terminated one (MPTES): the surface oxidized and cleaned silicon wafer was immersed in a $50 \mathrm{mM}$ solution of silane in anhydrous toluene at $75{ }^{\circ} \mathrm{C}$ for $24 \mathrm{~h}$. After silanization step, the samples were washed twice, sonicated for $10 \mathrm{~min}$ in anhydrous toluene, dried under nitrogen, and heated at $90{ }^{\circ} \mathrm{C}$ for $2 \mathrm{~h}$.

The MUA grafting was achieved after APTES deposition on silicon surfaces. First, MUA solution was activated using a mixture of EDC and NHS in ethanol during $90 \mathrm{~min}$ and then the APTES-modified silicon surfaces were placed in the activated solution of MUA for $90 \mathrm{~min}$, then washed twice in ethanol and dried under nitrogen. 
Gold nanoparticles preparation and deposition

Citrate-stabilized gold nanoparticles (GNPs) were prepared according to the standard method developed by J. Turkevich and co-workers and refined by G. Frens. Details on solution storage and particles dispersion are given in references $[11,12]$.

Freshly synthesized Au NPs were then deposited, with no further dilution, on the modified silicon surfaces for $2 \mathrm{~h}$ either using a gentle agitation or using a sonication bath (Elma, $90 \mathrm{~W}, 45 \mathrm{kHz}$ ). Silicon substrates were then washed twice in water and dried under nitrogen.

\section{Characterization techniques}

TEM Transmission electron microscopy measurements were performed using a JEOL JEM 1011 microscope operating at an accelerating voltage of $100 \mathrm{kV}$. The transmission electron microscopy (TEM) grids were prepared as follows: typically $1.5 \mathrm{~mL}$ of the solution was centrifuged at $10,000 \mathrm{rpm}$ (equivalent to 11,200 relative centrifugal forces) for $10 \mathrm{~min}$ to precipitate the particles. The colorless supernatant was discarded. The heavy residue was redispersed in a suitable volume of deionized water depending on the quantity of the residue. Of this redispersed particle suspension, $2 \mu \mathrm{L}$ was placed on a carbon-coated copper grid and dried at room temperature.

SEM Scanning electron microscopy images of the gold nanoparticles on the modified silicon wafers were obtained using a SEM FEG Hitachi SU-70 scanning electron microscope with a low voltage of $1 \mathrm{kV}$ and distance of $1.9-2.3 \mathrm{~mm}$; the secondary electron detector "in Lens" was used. The distribution of gold nanoparticles on the surfaces was established considering 1,000-1,500 particles.

Contact angle measurements Static water contact angles were measured at room temperature using the sessile drop method and image analysis of the drop profile. The instrument, which uses a CCD camera and an image analysis processor, was purchased from Krüss (Germany). The water (Milli-Q) droplet volume was $1 \mu \mathrm{L}$ and the contact angle $(\theta)$ was measured $5 \mathrm{~s}$ after the drop was deposited on the sample. For each sample, the reported value is the average of the results obtained on three droplets and the overall accuracy in the measurements was better than $\pm 5^{\circ}$.

XPS XPS analyses were performed with a PHOIBOS $100 \mathrm{X}$ ray photoelectron spectrometer from SPECS GmbH (Germany) with an monochromated $\mathrm{Al} \mathrm{K} \alpha \mathrm{X}$-ray source $(\mathrm{h} v=1,486.6 \mathrm{eV})$, operating at $10^{-10}$ Torr or less, in a "fixed analyzer transmission" analysis mode with a $7 \times 20 \mathrm{~mm}$ entrance slit, leading to a resolution of $0.1 \mathrm{eV}$. A $10 \mathrm{eV}$ pass energy for the survey scan and $10 \mathrm{eV}$ pass energy for the small regions were applied. The spectra were fitted using Casa XPS software (version 2.3.13, Casa Software, UK).

SERS Surface-enhanced Raman scattering spectra were recorded in the $500-1,900 \mathrm{~cm}^{-1}$ range on a modular Raman spectrometer (Model HL5R of Kaiser Optical Systems, Inc.) equipped with a high-powered near-IR laser diode working at $785 \mathrm{~nm}$. Before spectra acquisition, an optical microscope (Olympus; objective, $\times 50$ ) was used to focus the laser beam. Measurements were carried using an objective $\times 100$. The laser output power was $10 \mathrm{~mW}$, which corresponds to $\sim 1 \mathrm{~mW}$ on samples. For each spectrum, 30 acquisitions of $5 \mathrm{~s}$ were recorded to improve the signal-to-noise ratio. To ensure a representative characterization of surfaces, a minimum of three measurements were taken on different parts of the surface.

\section{Results and discussion}

Silane grafting and surface characterization

Gold nanoparticles are commonly immobilized on amineterminated layers, through electrostatic interaction or on thiol-terminated layers through covalent bonds. These two terminations were compared herein through the use of APTES or MPTES for silanization. Adding MUA on the APTES layer would lead to a longer chain terminated also by a thiol function, and thus enabling us to establish the influence of an additional parameter, namely, the hydrocarbon chain length. The three chemical modification of silicon surfaces are shown in Fig. 1. In a first stage, the efficiency of silane binding and further chemical grafting was investigated through contact angle measurements and XPS analysis of the functionalized silicon surfaces.

Contact angle measurements were performed to investigate the hydrophilic character of the three functionalized silicon surfaces. The pictures obtained with the CCD camera and the corresponding angles are shown in Fig. 2.

The initial clean silicon surface (Fig. 2a) was very hydrophilic and the contact angle $\left(<10^{\circ}\right)$ could not be measured due to drop spreading. This result confirms the efficiency of the applied cleaning procedure to remove the organic contaminants from the surface [23]. Upon APTES grafting (Fig. 2b), the measured contact angle was $83^{\circ}$, indicating a hydrophobic layer. This result is in agreement with previously observed values [18], the hydrophobic character, in apparent contradiction with the hydrophilic terminal amine functions, is probably due to the folding of amine terminal function to form $\mathrm{H}$ bonds with free silanol groups [24]. For the MPTES functionalization, the contact angle was lower than for 
Fig. 1 Schematic description of the three functionalized surfaces

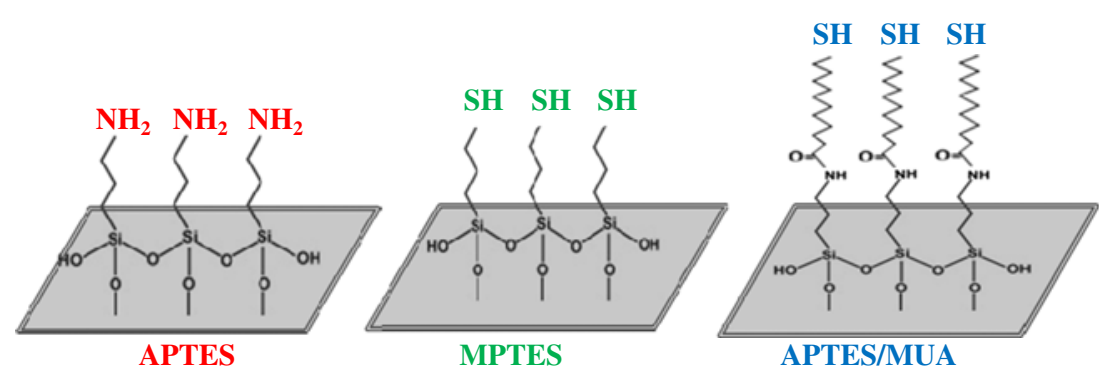

APTES-modified surfaces $\left(\sim 61^{\circ}\right)$. This higher hydrophilicity may be due to the lower tendency of sulfur to form hydrogen bonds; which could induce less folding of the silane chains. But another possibility, that will be discussed hereafter, on XPS data basis, is the incomplete coverage of silicon surface by MPTES; this would indeed lower contact angle values as the measured value will include a contribution of the hydrophilic-bare silicon oxide. Finally, upon grafting MUA on the APTES-modified layer, an even lower contact angle was recorded, $\sim 40^{\circ}$, suggesting a higher hydrophilic character of the resulting surfaces. The increase of hydrophilic character with chain length was observed likewise by Wasserman et al. upon assembling chloroalkylsilanes on silicon surfaces [25]. The most likely explanation is the increase of the layer crystallinity and organization due to the increase of Van der Waals interactions between alkyl chains [26]. This higher crystallinity would reduce the folding of the terminal groups, thus exhibiting the hydrophilic part of the grafted molecule on the upmost of the surface [27].
The modified silicon surfaces were also characterized by XPS analysis. The main XPS spectra are shown in Fig. 3. On all the survey spectra, in addition to the silicon and oxygen peaks arising from silicon wafers and the silica layer, carbon, nitrogen, and sulfur for APTES/MUA- and MPTES-modified surfaces were present. On the Si 2p peaks, shown in Fig. 3, two contributions were observed; the first one at $\sim 99 \mathrm{eV}$, arises from bulk silicon wafer $\mathrm{Si}^{0}$, and the second contribution at higher binding energy, $\sim 103 \mathrm{eV}$ is attributed to $\mathrm{Si}^{\mathrm{IV}}$ in both oxide layer and grafted silanes. The ratio $\mathrm{Si}^{\mathrm{ox}} / \mathrm{Si}^{0}$ was calculated to compare silane coverage, assuming the silica layer was similar in all cases. For the three surfaces, the values were very close, 0.64, 0.62, and 0.56, for APTES, MPTES, and APTES/MUA, respectively, suggesting similar average coverages with silanes, and not enabling a precise comparison of the amounts of grafted silanes.

The $\mathrm{N}$ 1s peaks included two contributions attributed to amine groups $\left[\mathrm{C}-\mathrm{NH}_{2}\right]$ (at $399.8 \mathrm{eV}$ ) and protonated amines $\mathrm{C}-\mathrm{NH}_{3}{ }^{+}$(at $401.6 \mathrm{eV}$ ). The shape of this peak was modified

Fig. 2 Static water contact angles pictures, and corresponding angles; a cleaned (oxidized) silicon wafer, b silicon-grafted APTES, c silicon-grafted MPTES, and $\mathbf{d}$ silicon-grafted APTES/MUA

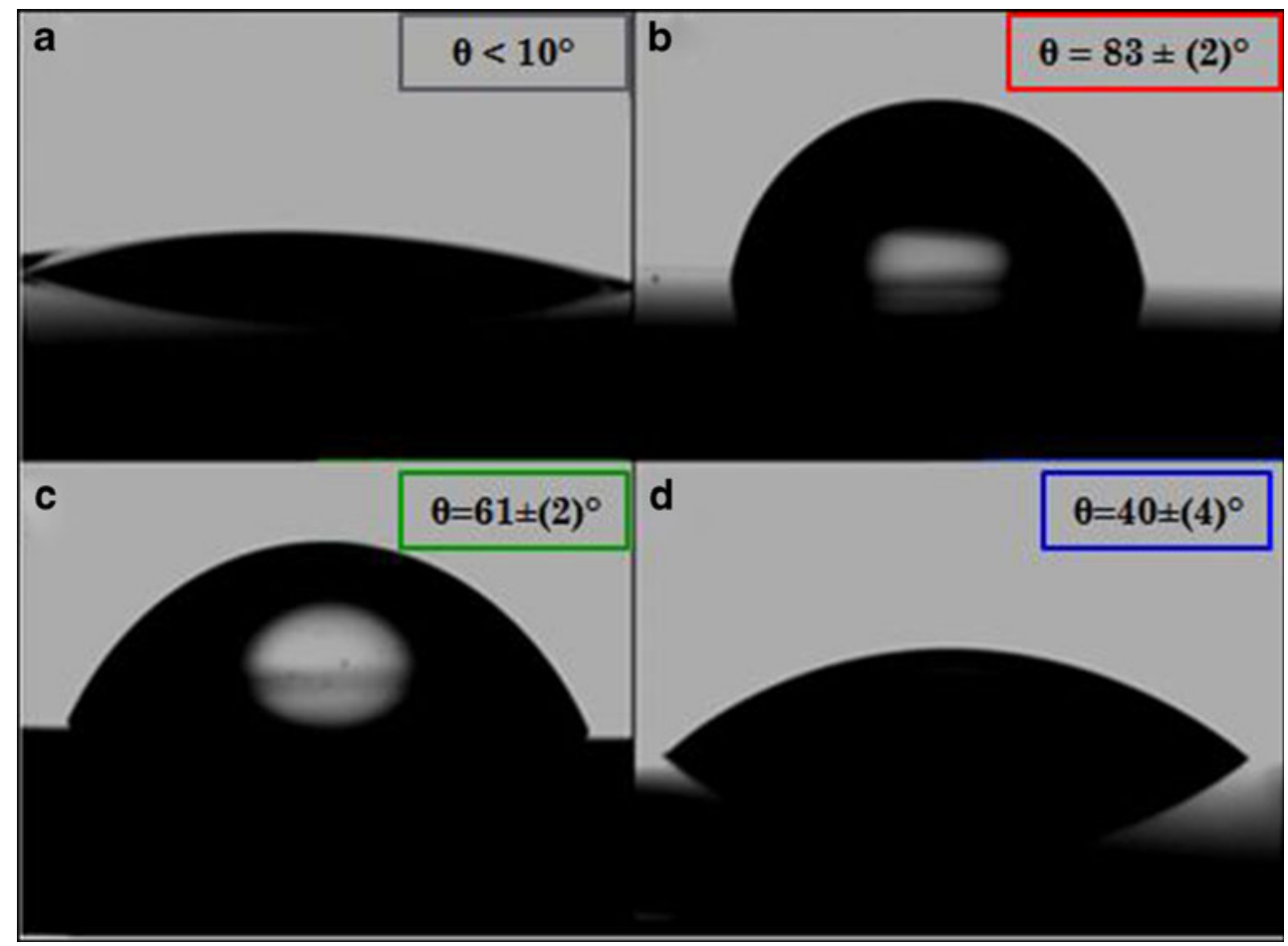



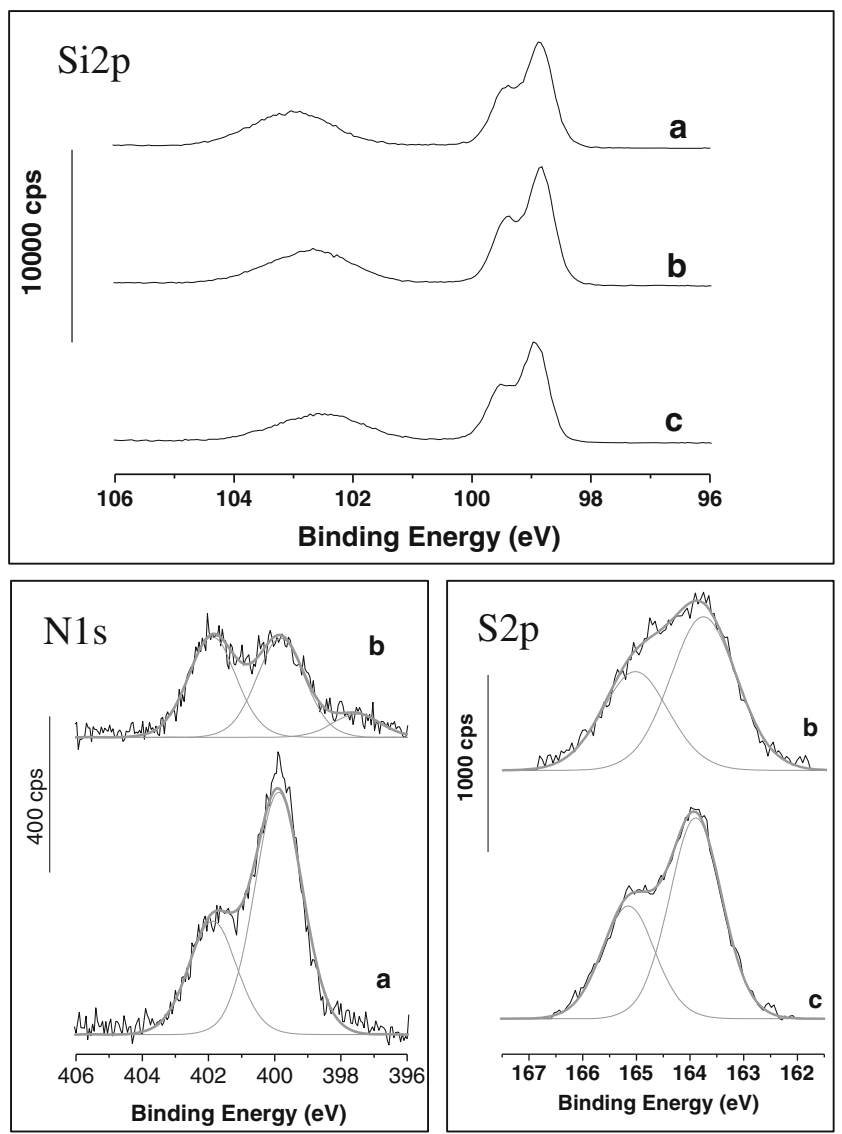

Fig. 3 XP spectra Si 2p, N1s, and S2p signals after APTES (a), APTES/ MUA (b), and MPTES (c) grafting on silicon surfaces

upon grafting MUA, but it is difficult to discriminate the contribution of amide nitrogen $[\mathrm{C}=\mathrm{O})-\mathrm{NH}]$ from the amine one [28]. The S 2 p spectra showed the $S 2 p$ doublet at 163.6 and $164.9 \mathrm{eV}$ for $\mathrm{S} 2 \mathrm{p}_{3 / 2}$ and $2 \mathrm{p}_{1 / 2}$, respectively, corresponding to the sulfur in the $\mathrm{SH}$ groups.

For the APTES/MUA-modified surface, the ratio S/N calculated using S 2p and N 1s peak was 0.24 . Thus, despite the large excess of MUA in solution, only ca. $24 \%$ of amine groups have reacted with the acid function of MUA. Therefore, thiol-end groups on these surfaces are "diluted" and four times less numerous than amine functions on APTESmodified surfaces. In addition, the integration of the S2p for both APTES/MUA and MPTES led to similar amounts of sulfur. Therefore, the use of MPTES would have led to a silane coverage four times lower than the use of APTES. This low value is not surprising as it was established than the terminal amine groups of APTES act as catalysts for silane reaction with silanols, thus enhancing the efficiency of grafting $[24,29]$. We confirmed these observations by performing the MPTES grafting using a small amount of ethylenediamine as catalyst. The XPS results (see Electronic supplementary material (ESM) 1) showed an increase of sulfur amount by a factor of 3.7, indicating a silane cover close to that obtained for APTES.

Gold nanoparticles deposition and characterization

Before interacting with the silane layers, the solution of gold nanoparticles was characterized by UV-visible spectroscopy. On this spectrum (see ESM), a narrow plasmon band was present at $525 \mathrm{~nm}$, indicating a homogeneous particle size of $\sim 13 \mathrm{~nm}[30,31]$. Gold nanoparticles size and distribution were also confirmed by TEM images (see ESM 1).

Figure 4 shows the Raman spectra of functionalized silicon surfaces before and after gold nanoparticles deposition. On the Raman spectrum of the functionalized silicon surface before the deposition of GNPs, the vibration bands of silanes could not be seen and only silicon signal was observed around 500 and $1,000 \mathrm{~cm}^{-1}$ for the silica layer. After gold nanoparticles deposition, a SERS effect is expected [32,33]. For the three functionalized surfaces, a Raman signal was observed, evidencing the grafting of gold nanoparticles $[34,35]$.
Fig. 4 Raman spectra obtained for gold nanoparticles deposited on silicon surfaces modified by APTES/MUA (a), APTES (b), and MPTES (c). d Silane layers prior to gold nanoparticles deposition

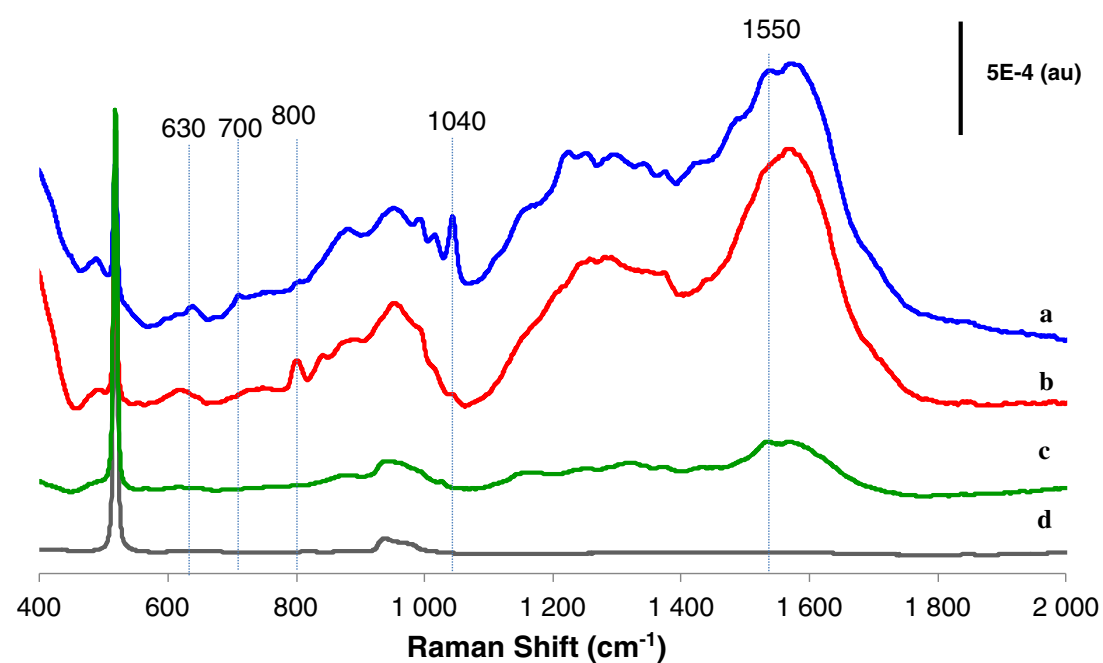


Fig. 5 SEM images of silicon surfaces after nanoparticle deposition without (a) and with (b and c) sonication on APTES, MPTES, and APTES/MUA

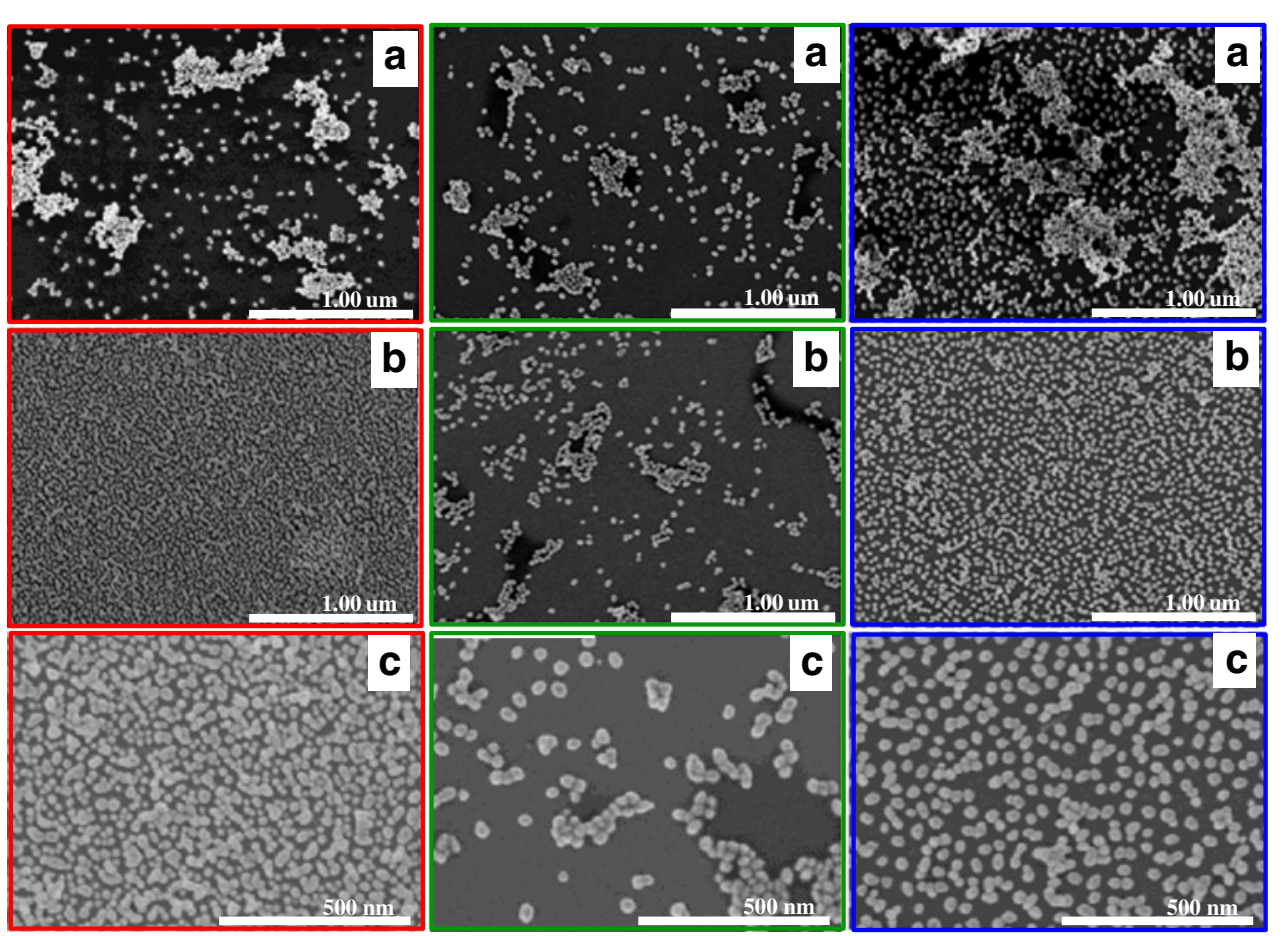

The main bands observed on SERS spectra arise from the characteristic bands of citrates and mainly of the carboxylate groups between 1,200 and $1,600 \mathrm{~cm}^{-1}[11,36]$. The $v(\mathrm{C}-\mathrm{C})$ arising from citrates and silane carbon chain formed massif of bands in the region $900-1,100 \mathrm{~cm}^{-1}$. Some differences, related to the different underlayer compositions, were observed and highlighted on the figure. On APTES/MUA, bands were present corresponding to $v(\mathrm{C}-\mathrm{S})_{\mathrm{G}}$ at $630 \mathrm{~cm}^{-1}$ and $v(\mathrm{C}-\mathrm{S})_{\mathrm{T}}$ at $700 \mathrm{~cm}^{-1}$, which is absent from the APTES spectrum. The intensities of the bands at 800 and $1,040 \mathrm{~cm}^{-1}$ arising from amine functions were modified upon MUA grafting on APTES and an additional band, amide II, appeared at $1,552 \mathrm{~cm}^{-1}$ [37].

Considering the citrates bands, the intensity of the signal was dependent on the system: While APTES- and APTES/ MUA-modified surfaces led to similar intensities; the MPTES-modified surfaces exhibited lower exaltation. This may be due to a lower coverage of GNPs as discussed below.

Figure 5 shows SEM images recorded after deposition of gold nanoparticles on APTES-, APTES/MUA-, and MPTESmodified silane surfaces. In Fig. 5a, the images obtained when nanoparticle deposition was carried out with a gentle agitation are shown. The highest coverage in gold nanoparticles was clearly observed on APTES/MUA-modified surfaces. However, for the three surface functionalization nanoparticles, aggregates are clearly observable with this deposition procedure.

When gold nanoparticles deposition was done under sonication, the largest nanoparticles aggregates were removed on both APTES and APTES/MUA surfaces. On these two systems, the dispersion was also clearly improved. The GNPs coverage on APTES was considerably increased. On MPTES layers, no significant improvement was observed using the sonication bath. The same results were obtained upon depositing the nanoparticles on the MPTES catalyzed by ethanediamine with or without sonication (images in the ESM 1). The sulfur density at silicon surface is therefore not a determining factor for this system.

Despite the improved dispersion and the removal of the largest aggregates, a closer inspection of the SEM images at higher magnification (Fig. 5c) showed the presence of small aggregates especially on APTES layers. To compare efficiently the dispersion on APTES and APTES/MUA layers, particle distributions were quantified for these two functionalizations and are presented as histograms in Fig 6.

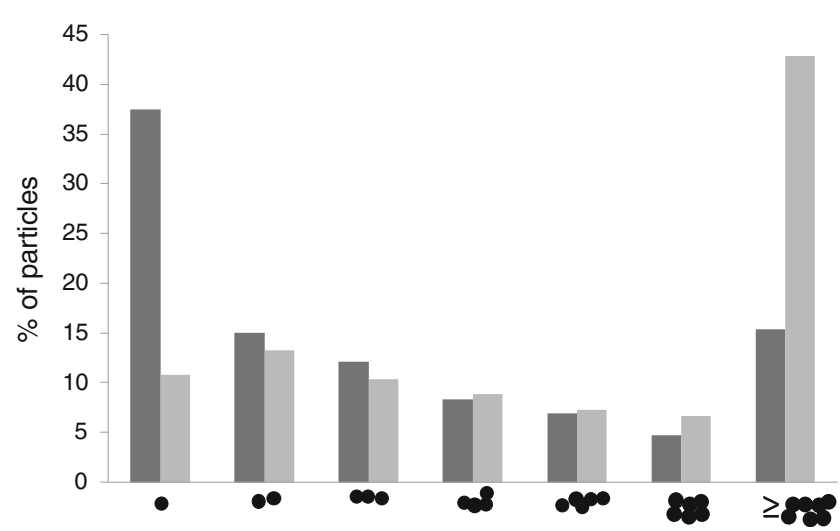

Fig. 6 Gold nanoparticles distribution on APTES/MUA (dark) and on APTES (bright)-modified layers 
The comparison of the aggregate size distribution for the two functionalized surfaces clearly showed that the APTES/ MUA functionalization allowed a better dispersion of the gold nanoparticles with a large fraction of isolated particles whereas, on the APTES functionalized surface, the nanoparticles formed higher amount of large aggregates. This better dispersion of gold nanoparticles on the MUA/APTES layer may result from the dilution of terminal functions on this system, $24 \% \mathrm{SH}$ on MUA/APTES vs $100 \% \mathrm{NH}_{2}$ on APTES. Indeed, similar behavior was observed using mixed thiol selfassembled monolayers to immobilize proteins: the dispersion was better when the terminal functions were diluted [38, 39]. The length of the alkyl chain induces also a better flexibility of the organic layer that probably favors nanoparticles binding compared to the MPTES layer [8].

\section{Conclusion}

We studied the organic layer influence on the grafting of gold nanoparticles on functionalized silicon substrates. To this aim, we grafted silanes terminated by either amine or thiol groups starting from APTES or MPTES. A third layer, also terminated by a thiol function, was constructed by covalent attachment of MUA on APTES-modified layers. XPS characterizations confirmed the successful grafting of APTES and MPTES on silicon surfaces. Upon adding MUA to the APTES-modified layers, only $24 \%$ of the amine functions reacted. Spherical gold nanoparticles were then deposited on these layers. SEM revealed that sonication during the deposition procedure limited the presence of aggregates on the surfaces. The higher coverage in gold nanoparticles was observed on APTES and APTES/MUA layers. SEM images also showed that gold nanoparticles dispersion was better on APTES/MUA than on APTES, with a larger fraction of isolated particles and few aggregates. This system likely benefits from both the dilution of terminal functions, $24 \% \mathrm{SH}$, and the flexibility of the organic layer.

Acknowledgments This work financially supported by the Agence Nationale de la Recherche "NArBIoS" project (ANR-DFG program; grant number: ANR-11-INTB-1013). The authors are grateful to ClaireMarie Pradier for her interest in the work.

Open Access This article is distributed under the terms of the Creative Commons Attribution License which permits any use, distribution, and reproduction in any medium, provided the original author(s) and the source are credited.

\section{References}

1. Sardar R, Funston AM, Mulvaney P, Murray RW (2009) Langmuir 25:13840-13851
2. Hoa XD, Kirk AG, Tabrizian M (2007) Biosens Bioelectron 23:151160

3. Daniel MC, Astruc D (2004) Chem Rev 104:293-346

4. Zheng J, Zhu Z, Chen H, Liu Z (2000) Langmuir 16:4409-4412

5. Haes AJ, Van Duyne RP (2004) Anal Bioanal Chem 379:920-930

6. Ghosh SK, Pal T (2007) Chem Rev 107:4797-4862

7. Luppa PB, Sokoll LJ, Chan DW (2001) Clin Chim Acta 314:1-26

8. Boujday S, Bantegnie A, Briand E, Marnet P-G, Salmain M, Pradier C-M (2008) J Phys Chem B 112:6708-6715

9. Hutter E, Fendler JH (2004) Adv Mater 16:1685-1706

10. Lalander CH, Zheng Y, Dhuey S, Cabrini S, Bach U (2010) ACS Nano 4:6153-6161

11. Morel A-L, Boujday S, Méthivier C, Krafft J-M, Pradier C-M (2011) Talanta 85:35-42

12. Morel A-L, Volmant R-M, Méthivier C, Krafft J-M, Boujday S, Pradier C-M (2010) Colloids Surf B Biointerfaces 81:304-312

13. Zhong Z, Patskovskyy S, Bouvrette P, Luong JHT, Gedanken A, Phys J (2004) Chem B 108:4046-4052

14. Aureau D, Varin Y, Roodenko K, Seitz O, Pluchery O, Chabal YJ (2009) J Phys Chem C 114:14180-14186

15. Khire VS, Lee TY, Bowman CN (2007) Macromolecules 40:56695677

16. McNally H, Janes DB, Kasibhatla B, Kubiak CP (2002) Superlattice Microst 31:239-245

17. Gooding JJ, Ciampi S (2011) Chem Soc Rev 40:2704-2718

18. Aissaoui N, Bergaoui L, Landoulsi J, Lambert JF, Boujday S (2012) Langmuir 28:656-665

19. Kim J, Cho J, Seidler PM, Kurland NE, Yadavalli VK (2010) Langmuir 26:2599-2608

20. Pasternack RM, Rivillon Amy S, Chabal YJ (2008) Langmuir 24: 12963-12971

21. Howarter JA, Youngblood JP (2006) Langmuir 22:11142-11147

22. Kaminska A, Inya-Agha O, Forster RJ, Keyes TE (2008) Phys Chem Chem Phys 10:4172-4180

23. Mittal KL (1979) Surface contamination. In: Mittal KL (ed). Springer, New York, pp 3-45

24. Kanan SM, Tze WTY, Tripp CP (2002) Langmuir 18:6623-6627

25. Wasserman SR, Tao YT, Whitesides GM (1989) Langmuir 5:10741087

26. Love JC, Estroff LA, Kriebel JK, Nuzzo RG, Whitesides GM (2005) Chem Rev 105:1103-1169

27. Vallée A, Humblot V, Al Housseiny R, Boujday S, Pradier C-M (2013) Colloids Surf B Biointerfaces 109:136-142

28. Mercier D, Boujday S, Annabi C, Villanneau R, Pradier C-M, Proust A (2012) J Phys Chem C 116:13217-13224

29. Blitz JP, Murthy RSS, Leyden DE (1987) J Am Chem Soc 109:71417145

30. Turkevich J, Hillier J, Stevenson PC (1951) Discuss Faraday Soc 11: 55

31. Seitz O, Chehimi MM, Cabet-Deliry E, Truong S, Felidj N, Perruchot C, Greaves SJ, Watts JF (2003) Coll Surf A: Physicochem Eng Aspects 218:225-239

32. Grabar KC, Freeman RG, Hommer MB, Natan MJ (1995) Anal Chem 67:735-743

33. Chumanov G, Sokolov K, Gregory BW, Cotton TM (1995) J Phys Chem 99:9466-9471

34. Moskovits M (2005) J Raman Spectrosc 36:485-496

35. Tian ZQ (2005) J Raman Spectrosc 36:466-470

36. Bantz KC, Nelson HD, Haynes CL (2012) J Phys Chem C 116:35853593

37. Richard Allen N (2001) Interpreting infrared, Raman, and nuclear magnetic resonance spectra. Academic, San Diego, pp 143-148

38. Briand E, Gu C, Boujday S, Salmain M, Herry JM, Pradier CM (2007) Surf Sci 601:3850-3855

39. Thébault P, Boujday S, Sénéchal H, Pradier C-M (2010) J Phys Chem B 114:10612-10619 